\title{
We Hommage à Jean Laplanche
}

Christophe Dejours ${ }^{[1]}$

ean Laplanche a consacré sa vie à la psychanalyse. L'œeuvre de Freud il l'a d'abord et avant tout travaillée et fouillée en philosophie, avec la maitrise et le raffinement d'un penseur qui était dépositaire de ce que la tradition philosophique française a de meilleur. Et il est juste d'honorer d'abord ce que l'on doit à son œuvre de philosophe : l'extraction du tissu de concepts sur lesquels repose l'architecture de la métapsychologie freudienne. Ce magnifique travail qu'il a accompli avec Jean Bertrand Pontalis est, peut-être, la contribution la plus importante qui ait été apportée à la pérennité de l'œuvre de Freud, depuis Freud. Jean Laplanche était de surcroît un praticien de la psychanalyse, et un maître dans ce compagnonnage qui consiste à transmettre les savoir-faire du métier de psychanalyste dont beaucoup d'entre nous avons eu la chance de profiter.

Mais, comme il le disait lui-même, il avait un deuxième métier. Et tout le monde, alors, de penser à son métier de viticulteur. Ce n'était pas ce qu'il voulait, de cette façon indiquer. Son second métier c'était celui de traducteur. Traduire, pour lui, ce n'était pas seulement faire ouvre de germaniste traduisant des textes de l'allemand au français. Car c'est bel et bien de ce travail de traduction que Jean Laplanche a tiré la Théorie de la Séduction Généralisée pour rendre compte de la sexualité humaine. Traduire Freud c'était donc aussi prendre l'œuvre de Freud comme un message. « Le message freudien " contenu dans ses ouvres complètes était alors à comprendre comme un message, oh combien! “ compromis », laissé par Freud aux lecteurs que nous sommes. Ainsi faut-il sans doute reconnaître que Freud lui-même, a été l'un des grands séducteurs du $\mathrm{XX}^{\mathrm{e}}$ siècle, non pas au sens ironique du terme, mais au sens de ce qui caractérise la position de l'adulte par rapport à celle de l'enfant. Freud adulte séducteur - a fait, de millions de lecteurs, des enfants herméneutes. Et parmi eux il a séduit un enfant particulièrement génial : Jean Laplanche. Entouré, il est vrai, d'un groupe auquel nous devons aussi dire notre gratitude : Janine Altounian, André

[1] Président du Conseil Scientifique de la Fondation Jean Laplanche.

Article disponible sur le site nttp://WWW.psycho-cIInIque.org ou nttp://ax.dol.org/10.1051/psyc/201234243 
Bourguignon, Pierre Cotet, Alain Rauzy, François Robert et quelques autres, Jean Laplanche a œuvré une bonne partie de sa vie à déchiffrer le texte freudien.

Mais à son tour, l'œuvre de Jean Laplanche, et sa Théorie de la Séduction Généralisée, forment ensemble un nouveau message à traduire, pour ses lecteurs et ses élèves. Et nous sommes désormais nombreux à travers le monde à nous trouver pris dans le travail d'herméneutes du message laplanchien. Jean Laplanche, il faut le reconnaître, était un grand séducteur devant l'éternel... et devant la communauté scientifique. Car pour lui la “ situation anthropologique fondamentale » avec, en son centre la séduction, plus universelle assurément que le complexe d'Edipe - est l'une des conséquences essentielles de la découverte psychanalytique. C'est la raison pour laquelle la psychanalyse est d'abord et avant tout, comme il est arrivé à Laplanche de le dire : "La théorie de la sexualité humaine » ou encore la seule véritable théorie constituée et cohérente de la sexualité humaine.

Cette "passion " pour la sexualité humaine qui aboutit à la démonstration de son importance ou mieux encore, de sa centralité dans la vie psychique et la culture tout entière, ne conduit nullement à la conclusion que tout en ce monde serait sexuel. Pas du tout ! Jean Laplanche a accordé beaucoup d'attention et de temps à ce qui ne ressortit pas au sexuel, dans la société d'un côté, dans la vie biologique et l'autoconservation de l'autre. La Théorie de la Séduction Généralisée est tout sauf un pansexualisme.

Le corps, les instincts, les besoins du corps d'abord qui appellent en retour le soin, le care dirait-on aujourd'hui. Aucun d'entre nous ne deviendrait adulte s'il ne profitait du care. Et aucun adulte, même, ne pourrait se passer du care lorsqu'il est malade ou lorsqu'il va vers sa mort. J'y insiste pour pouvoir dire quelques mots sur ceux qui ont accompagné Jean pendant ses années de maladie. Nadine, son épouse, d'abord, mais aussi ses amis les plus proches, en particulier Monsieur et Madame Moati, grâce auxquels les archives de Jean Laplanche seront mises incessamment à la disposition des chercheurs.

Même si c'est la tristesse de la mort de Jean Laplanche, survenue le 6 mai 2012, à l'âge de 87 ans, qui a réuni tant de gens au cimetière de Pommard, je ne voudrais pas finir sur le spectre des ténèbres. La mort n'est pas toujours le terme indépassable de la vie. De Socrate nous tenons que la lutte pour l'immortalité n'est pas vaine. Il y a deux façons de survivre à la mort. Par la création et par la procréation. Pour ce qui est de cette dernière, Jean et Nadine n'ont pas eu la chance de pouvoir donner naissance à des enfants. Mais ils ont pour une large part élevé un neveu : Olivier et assurément ils l'ont aimé comme des parents, ainsi que ses deux fils.

Quant à ce qu'il en est de la création, l'œuvre de Jean Laplanche n'est rien si ce n'est un monument. Pas au sens du Palais Garnier, ni des châteaux de Ludwig de Bavière à Füßen. Bien au contraire. Et s'il fallait choisir un style architectural pour caractériser le monument laplanchien, il me semble que celui qui conviendrait le mieux serait le style Directoire. 
Car parmi les œuvres de Laplanche qui résistent à la mort, il faut, je crois, faire aussi une place à Pommard. Non seulement à la construction patiente de ce domaine viticole, le plus grand vignoble d'un seul tenant de toute la Bourgogne, il faut faire une place non seulement à l'élaboration d'un cru et à un art de la vinification que nombreux lui reconnaissent, mais je pense ici aux deux châteaux : à celui de Marey-Monge

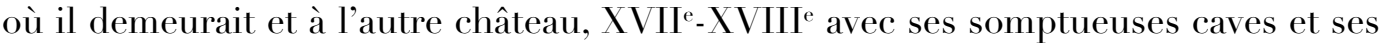
dépendances. Jean, mais Nadine y a beaucoup donné d'elle aussi, ont entretenu, restauré et embelli parfois ces châteaux, avec un talent et un savoir-faire grâce auxquels de belles pierres du patrimoine de France sont aujourd'hui transmises à la postérité. C'est une partie de leur ouvre et je souhaite aussi la célébrer.

L'autre part de création par laquelle Jean Laplanche se survivra à lui-même, c'est bien sûr son ouvre de savant. Mais il n'y aurait pas de surmontement de la mort pour reprendre un des termes prisés de Laplanche - s'il n'y avait des livres pour lui survivre. Et il n'y a pas de livre sans éditeur. C'est pourquoi dans cet hommage il convient aussi de remercier les Presses Universitaires de France, de rappeler à cette occasion la mémoire de Michel Prigent, et dire nos voux à ses successeurs, à Monique Labrune et à toute leur équipe pour avoir rassemblé l'œuvre de Laplanche et avoir réalisé le magnifique chantier presque achevé de l'édition complète des oeuvres de Freud en français.

Dans son travail Jean Laplanche a toujours cherché le débat, avec ses amis de l'Association Psychanalytique de France d'abord, avec ses élèves à travers le monde ensuite, mais aussi avec le séminaire dit “Séminaire de Pommard " qui rassemblait ceux qui étaient, en définitive, des amis précieux, à commencer par Jean-Louis Brenot le plus proche parmi tous et Maddy, Gilbert Diebold, François Auger, Jacky Rigaud, Bertrand Barré et quelques autres encore parce qu'ils ont beaucoup compté pour lui.

Et il faut remercier l'Institut de France et son chancelier Monsieur Gabriel de Broglie, pour l'avoir accueillie et pour la contribution qu'ils apportent à la “ Fondation Jean Laplanche-Nouveaux fondements pour la psychanalyse ", que Jean et Nadine ont voulue, afin d'apporter à leur façon, une participation durable au débat sur la psychanalyse et sur l'œuvre de Freud, en France et à travers le monde. Et c'est l'occasion pour nous de dire que les membres de cette Fondation et les membres de son Conseil Scientifique - José Carlos Calich de Porto Alegre, Adriana Cinello de Madrid, Gilbert Diebold de Besançon, Udo Hock de Berlin, Jonathan House de New York, Alberto Luchetti de Rome, Marcelo Marquès de Paris, Francis Martens de Bruxelles et Hélène Tessier d'Ottawa - feront en sorte d'honorer et Freud et Laplanche et de permettre que l'œuvre de Laplanche continue de catalyser le débat entre psychanalystes et le débat entre la psychanalyse et les autres disciplines.

Du 3 au 5 juillet 2012 se sont tenues les Journées internationales Jean Laplanche à Paris dans les salons de l'Hôtel del Duca qui appartient à l'Institut de France. Ces Journées ont lieu tous les deux ans depuis une vingtaine d'années. Pour la première 
fois elles se sont déroulées sans la présence de Jean Laplanche, mais l'esprit de ces rencontres a été respecté. Le thème était : "Théorie de la séduction généralisée et pratique psychanalytique » et avait été choisi en accord avec Jean Laplanche.

En juillet 2014 des Journées Jean Laplanche se tiendront à Cerisy la Salle. Le thème général en sera : “La séduction à l'origine ; l'œuvre de Jean Laplanche ». Elles seront organisées à l'initiative de l'Association Psychanalytique de France, en partenariat avec la Fondation Jean Laplanche. Les trois volets de ce colloque : les moments théoriques de l'œuvre, les incidences de la théorie sur la pratique analytique et la psychanalyse “ exportée » (ou “ hors cure »), articuleront les interventions de façon à permettre la discussion la plus large entre analystes et chercheurs d'autres disciplines et d'autres cultures linguistiques.

D'autres manifestations sont en cours d'organisation, en France et à l'étranger, qui autorisent à penser que la pensée de Jean Laplanche contribuera à faire vivre le débat sur la place qui revient à la sexualité dans la théorie psychanalytique et dans les oeuvres de la culture. 